\title{
PERFORMANCE OF DIFFERENT MUNGBEAN (VIGNA RADIATA (L.) WILCZEK) GENOTYPES AGAINST MEGALUROTHRIPS DISTALIS (THYSANOPTERA: THRIPIDAE) IN THE AGRO- ECOLOGICAL ZONE OF BHAKKAR, PAKISTAN
}

\author{
NADEEM, M. $.^{1,2^{*}}-$ SHAHZAD, M. F. ${ }^{1}-$ HuSSAIN, K. ${ }^{2}$ \\ ${ }^{I}$ Faculty of Agriculture, Gomal University, Dera Ismail Khan, Pakistan \\ ${ }^{2}$ Arid Zone Research Institute, Bhakkar, Pakistan \\ *Corresponding author \\ e-mail: nadeem_agrarian@yahoo.com; +92-343-799-9789 \\ (Received 25 $5^{\text {th }}$ Jul 2020; accepted 22 ${ }^{\text {nd }}$ Oct 2020)
}

\begin{abstract}
The experiment was conducted at the Arid Zone Research Institute (AZRI), Bhakkar, Pakistan during 2018 and 2019 on different mungbean genotypes to find a resistant to thrips and to determine the impact of weather factors on the Megalurothrips distalis population. In 2018 twenty-five mungbean genotypes were screened against $M$. distalis. During 2019 three genotypes each of resistant (13TM-04, AZRI-06, NM-16), susceptible (Dera-M, Sawat-I, NM-98) and intermediate reaction (NM-06, 12TM-03, NM-11) towards thrips infestation were chosen for final screening based on per flower thrips population density at 4 days intervals commencing from the first appearance of thrips up to maturity stage of the crop. Maximum HPSI, $15 \%$ was recorded in Dera $-\mathrm{M}$ while the minimum was recorded on 13TM-04 and NM-16 with $8 \%$ of each. Yield and the yield contributing characteristics were also assessed from which NM-16 and 13TM-04 exhibited statistically similar results and produced the highest yields at 935 and $902.8 \mathrm{~kg} / \mathrm{ha}$ respectively. During both study years and on cumulative basis thrips population fluctuation had positive results and showed a significant correlation with maximum and average temperature. The average number of thrips during 2018, 2019 as well as both years combined showed a non-significant correlation with minimum temperature while negative and highly significant correlation was observed with average relative humidity and rainfall. Maximum temperature was the most important factor which contributed maximum i.e. $51.1 \%$ and $55.8 \%$ role followed by rainfall which showed $14.8 \%$ and $4 \%$ role during 2018 and 2019 respectively in the fluctuation of thrips population.
\end{abstract}

Keywords: effect, weather factors, thrips population, HPSI (\%), mungbean yield

\section{Introduction}

Mungbean, Vigna radiata (L.) Wilczek, is an important pulse crop grown in Pakistan. It is able to grow efficaciously in rain fed regions (Anjum et al., 2006). It is a short duration and might face up to unfavorable environmental situations therefore is broadly distributed all around the world. It is the most preferred by the farmer due to high protein content and is easily adopted in the drier and warmer climates (Ratnasekera and Subhashi, 2015; Yaqub et al., 2010).

The yield and quality of mungbean is affected by different biotic (pest and diseases), abiotic (temperature, rainfall, relative humidity etc.) and phenological factors (flower and fruit drop). Among biotic factors insect pests often cause serious threat to mungbean production and increase the cost of production. Insect pests incidence significantly reduces mungbean yield and quality (Malik et al., 1994). Among the insect pests of mungbean thrips had gained major importance. The nature and extent of damage caused by thrips in mungbean varies in distinctive climatic areas because of various agroclimatic conditions. Thrips is the regular, early season and most important 
insect pest of mungbean which inflict heavy yield losses (Hossain et al., 2004). Flower shedding to the extent of 40-89\% has been pronounced in mungbean (Kaul et al., 1976; Sinha, 1977). Flower infestation by thrips results in flower drop, pods deformation, grain quality deterioration and ultimately excessive yield reduction. Yield losses are estimated to be around 65\% in mungbean due to thrips attack (Indiati, 2004).

The endurance, growth and reproductive capability of insect pests depends on the climatic factors (Morsello et al., 2014; Rosado et al., 2015). An insect development is influenced by the prolonged duration of low, high or sudden change in temperatures (Iqbal et al., 2010). Weather parameters have significant impact on thrips population (Pedigo, 2004). Different weather factors have positive association for the development and seasonal incidence of thrips (Soni and Dhakad, 2016). Economic loss due to thrips could be reduced by collecting such prediction information (Shuaib, 2004). Such studies are helpful in the establishment of predictive models of thrips attack on mungbean, so that the farmers can determine the correct time to start pest control (Da Silva et al., 2017; Herms, 2004; Rosado et al., 2015)

The varietal development in mungbean focused namely on the selection from germplasm. Several resistant donors for thrips were identified and used to transfer gene(s) for pest resistance. As a result, a number of high yielding genotypes with limited thrips tolerance were developed through interspecific hybridization. There is a need to intensify research for thrips tolerance through integration of desirable alleles from secondary and tertiary gene pool.

Due to the damage significance caused by thrips to the mungbean crop, an attempt was made to sort out the correlation of climate factors with thrips population in mungbean crop under agroclimatic conditions of Bhakkar to predict seasons of abundance. The present study objective was to determine resistant genotype of mungbean against thrips and fluctuation of thrips population in mungbean in relation to weather factors. The present study would be helpful for determining the peak period of thrips attack which in turn may be supportive in developing better thrips management strategy.

\section{Materials and methods}

Present research was conducted during the years 2018 and 2019 to find out the resistant genotype of mungbean and to work out the weather role in thrips population fluctuation. Twenty-five genotypes of mungbean were sown at AZRI, Bhakkar, Pakistan on the $10^{\text {th }}$ May, 2018. Plot size was maintained $5 \times 2.4 \mathrm{~m}$ with $30 \mathrm{~cm}$ row to row and $10 \mathrm{~cm}$ plant to plant distance. For further investigations three genotypes resistant, susceptible and showing intermediate reaction to thrips infestation were chosen. Thus, in total, nine genotypes were chosen from preliminary screening trial of 2018 based on per flower thrips population density. Sowing of the trial was done on May 10, 2019 in the same experimental area following Randomized Complete Block Design (RCBD) replicated thrice with the same plot size.

During both research years no plant protection measures were applied. Recommended agronomic practices were applied as and when required. From randomly selected five plants thrips population data was taken avoiding border rows from each treatment and three opened flowers of each randomly selected plant were observed at 4 days interval commencing from the first appearance of thrips up to maturity stage of the crop. Thus, from each plot a total of 15 flowers were observed for collection of 
thrips data. The collected flowers were immediately opened on white paper board and thrips numbers were recorded by counting number of thrips (through the use of magnifying lenses). The average thrips population on per flower basis for each genotype was determined by

$$
X=\frac{X 1+X 2+X 3_{\operatorname{manmmax}}+X 14+X 15}{15}
$$

where: $X=$ mean number of thrips flower ${ }^{-1}$ and $X_{1}+X_{2}+X_{3}+, \quad \cdots$ $+\mathrm{X}_{14}+\mathrm{X}_{15}=$ number of observed flowers.

Mungbean genotypes were classified into three categories based on attack of mean number of thrips per flower:

3 - 3.99 thrips/flower $=$ Resistance; $4-4.99$ thrips/flower $=$ Intermediate Resistance; $5-6$ and above thrips/flower $=$ Susceptible.

\section{Host plant susceptibility indices (HPSIs)}

The HPSI, based on thrips infestation at different genotypes of mungbean was determined using the following formula:

$$
\operatorname{HPSI}(\%)=100-\frac{B-A}{B} \times 100
$$

where $\mathrm{A}=$ thrips population in individual mungbean genotype and $\mathrm{B}=$ thrips population in all genotypes of mungbean on average per leaf or per flower basis (Ali and Farooq, 2019).

At maturity from five sample plants total number of flowers, flower shedding, total pods and deformed pods were recorded. Then their average number per plant data was calculated. After harvesting of the crop No. of seeds/pod was recorded from the five randomly selected pods. Then from their mean value average number of seed per pod was calculated. Weight of seeds per plant was also calculated from total number of seeds obtained from five randomly selected plants from each plot which were properly sun-dried and their weights were recorded. Then from their mean value weight of seeds per plant was calculated. 100 seed of each selected genotype was separately weighed and data was recorded. After harvesting crop was sun-dried and threshed. Seeds were properly sun-dried and their weights were recorded. Yield per plot was converted in to yield per hectare (Sagar et al., 2017). To find out the significance difference between the different treatments the data on aforementioned parameters were statistically analyzed. By using analysis of variance techniques (Steel and Torrie, 1997) thrips infestation data were analyzed and means were compared by (post-hoc test) using least significant difference test (LSD) at 5\% significance level.

Meteorological data related to atmospheric temperature (maximum, minimum and average temperature), average R.H and total rainfall was obtained from the meteorological observatory of AZRI, Bhakkar for the relevant months and were correlated with per flower thrips population of various selected genotypes of mungbean with the objective to determine the impact of different abiotic factors on thrips population. These factors were processed for simple correlation (Steel et al., 1997) between population of thrips and abiotic factors and the factors which showed significant correlation with the thrips population were computed for multiple linear regression analyses of variance with the objective to find the percent role of these 
factors individually as well as in their possible combinations through steps. The data obtained during 2018 and 2019 were transformed to square root transformations and were statistically analyzed. By using Multiple Linear Regression Equation, the combined effect of the abiotic factors on thrips population during 2018 and 2019 were calculated.

\section{Results}

Varietal performance of different mungbean genotypes against Megalurothrips distalis during 2018

The results (Table 1) showed the mean number of thrips per flower population during 2018 and 2019. It is evident from the results that during 2018 on the basis of mean population of thrips maximum thrips population were observed on Sawat-1 and Dera-M which were at par with each other with 6.03 and 6.00 thrips per flower followed by NM-98 with 5.57 thrips per flower. The minimum 3.07 thrips/flower population was recorded in 13TM-04 which was statistically at par with the genotypes NM-16 and AZRI-06 which showed 3.27 and 3.40 thrips per flower population each during 2018. The genotypes NM-11, 12TM-03 and NM-06 showed 4.77, 4.67 and 4.53 thrips per flower population, respectively which were at par with each other. On the basis of mean number of $M$. distalis population during 2018 all the mungbean genotypes were categorized in descending order as follows: Sawat-1 > Dera-M > NM-98 > TM-1626 > TM-1611 > TM-1609 > Chakwal-06 > Inqilab-M > TM-1607 > TM-1615 > TM-1601 > NM-11 > 12TM-03 > NM-06 > Bahawalpur mung-17 > NCM-13 > Sona-M > NM-92 > TM-1418 > TM-1627 > 13TM-14 > 09TM-11 > AZRI-06 > NM-16 and 13TM-04.

\section{Varietal performance of different genotypes against thrips during 2019}

From the results of preliminary screening trial, three highly susceptible genotypes (Dera-M, Sawat-1 and NM-98) three intermediate response genotypes (NM-11, NM-06 and 12TM-03) and three with the lowest thrips population showing resistant response (13TM-04, NM-16 and AZRI-06) were chosen for final screening trial during 2019. The results (Table 1) showed that maximum number of thrips 6.27 per flower was observed on Dera-M followed by Sawat-1 and NM-98 with 5.90 and 5.73 thrips per flower respectively which were statistically at par with each other. While minimum 3.50 thrips per flower population was recorded in 13TM-04 followed by NM-16 with 3.53 thrips per flower which was statistically similar with AZRI-06 with 3.87 thrips per flower. The genotype 12TM-03 showed 4.47 thrips per flower which was statistically similar with NM-06 with 4.73 thrips per flower followed by NM-11 which showed 4.87 thrips.

\section{Host plant susceptibility indices (HPSI\%)}

The results (Table 2) revealed the HPSI (\%), based on per flower thrips population on different genotypes of mungbean during 2018 and 2019. The genotype Dera-M showed maximum 15\% HPSI during 2018 and 2019 on per flower basis followed by Sawat-1 which showed 15\% HPSI\% during 2018 and 14\% during 2019. While NM-98 showed 13\% HPSI during both years. These three genotypes were found comparatively susceptible to thrips infestation. Genotypes 12TM-03 showed 11\% and 10\% HPSI\% during 2018 and 2019 respectively while NM-11 showed 11\% HPSI each during both years. NM-11 showed 12\% during 2018 and 11\% HPSI during 2019. The minimum 7\% 
HPSI was recorded in 13TM-04 during 2018 while it was $8 \%$ during 2019 followed by NM-16 which showed $8 \%$ HPSI of each during both years. While AZRI-06 showed $8 \%$ during 2018 and 9\% HPSI during 2019.

Table 1. Mean comparison of the data regarding thrips population on various genotypes of Mungbean, at different dates of observations during 2018 and 2019

\begin{tabular}{c|c|c}
\hline \multirow{2}{*}{ Genotypes } & \multicolumn{2}{|c}{ Thrips per flower during } \\
\cline { 2 - 3 } & $\mathbf{2 0 1 8}$ & $\mathbf{2 0 1 9}$ \\
\hline Dera-M & $6.00 \mathrm{a} *$ & $6.27 \mathrm{a}$ \\
Sawat-1 & $6.03 \mathrm{a} *$ & $5.90 \mathrm{~b}$ \\
NM-98 & $5.57 \mathrm{~b} *$ & $5.73 \mathrm{~b}$ \\
TM-1626 & $5.43 \mathrm{bc}$ & \\
TM-1611 & $5.40 \mathrm{bc}$ & \\
TM-1609 & $5.37 \mathrm{bc}$ & \\
Chakwal-06 & $5.33 \mathrm{bc}$ & \\
Inqilab-M & $5.13 \mathrm{~cd}$ & \\
TM-1607 & $4.87 \mathrm{de}$ & \\
TM-1615 & $4.87 \mathrm{de}$ & \\
TM-1601 & $4.83 \mathrm{def}$ & $4.87 \mathrm{c}$ \\
NM-11 & $4.77 \mathrm{efg} * *$ & $4.47 \mathrm{~d}$ \\
$\mathbf{1 2 T M - 0 3}$ & $4.67 \mathrm{efgh} * *$ & $4.73 \mathrm{~cd}$ \\
NM-06 & $4.53 \mathrm{efgh} * *$ & \\
Bhawalpur M-17 & $4.50 \mathrm{fgh}$ & \\
NCM-13 & $4.43 \mathrm{gh}$ & \\
Sona-M & $4.37 \mathrm{~h}$ & \\
NM-92 & $3.93 \mathrm{i}$ & \\
TM-1418 & $3.83 \mathrm{ij}$ & \\
TM-1627 & $3.70 \mathrm{ijk}$ & \\
$\mathbf{1 3 T M - 1 4}$ & $3.70 \mathrm{ijk}$ & $3.57 \mathrm{e}$ \\
09TM-11 & $3.50 \mathrm{jkl}$ & \\
AZRI-06 & $3.40 \mathrm{klm} * * *$ & $\mathrm{f}$ \\
NM-16 & $3.27 \mathrm{~lm} * * *$ & \\
$\mathbf{1 3 T M - 0 4}$ & $3.07 \mathrm{~m} * * *$ & \\
LSD value @5\% & 0.36 & \\
\hline
\end{tabular}

Means sharing similar letters are not significantly different by LSD test at $\mathrm{P}=0.05$

$*$ = Susceptible genotypes

** = Intermediate resistant genotypes

$* * *=$ Resistant genotypes

The results (Table 2) showed the HPSI (\%) on cumulative basis of both study years 2018-19 which showed that maximum HPSI 15\% was recorded in Dera-M followed by Sawat-1 and NM-98 with $14 \%$ and 13\% respectively which showed that these three genotypes are more susceptible to thrips infestation. While 12TM-03, NM-06 and NM11 showed $11 \%$ HPSI of each genotype which are found intermediate response. The minimum HPSI was recorded on 13TM-04 and NM-16 with $8 \%$ of each followed by AZRI-06 which showed9\% HPSI which showed the resistant response to thrips infestation on per flower basis. 
Table 2. Host plant susceptibility indices (\%) based on mean percentage of thrips on different genotypes of mungbean during 2018 and 2019

\begin{tabular}{c|c|c|c}
\hline \multirow{2}{*}{ Genotypes } & \multicolumn{3}{|c}{ Host plant susceptible indices (\%) based on per flower during } \\
\cline { 2 - 4 } & $\mathbf{2 0 1 8}$ & $\mathbf{2 0 1 9}$ & Cumulative of 2018 and 2019 \\
\hline Dera-M & $15 \%$ & $15 \%$ & $15 \%$ \\
Sawat-I & $15 \%$ & $14 \%$ & $14 \%$ \\
NM-98 & $13 \%$ & $13 \%$ & $13 \%$ \\
$\mathbf{1 2 T M - 0 3}$ & $11 \%$ & $10 \%$ & $11 \%$ \\
NM-06 & $11 \%$ & $11 \%$ & $11 \%$ \\
NM-11 & $12 \%$ & $11 \%$ & $11 \%$ \\
AZRI-06 & $8 \%$ & $9 \%$ & $9 \%$ \\
NM-16 & $8 \%$ & $8 \%$ & $8 \%$ \\
13TM-04 & $7 \%$ & $8 \%$ & $8 \%$ \\
\hline
\end{tabular}

\section{Response of different mungbean genotypes against thrips attack}

Response of different mungbean genotypes against thrips was assessed by comparing the total flowers, flower shedding, total pods and deformed pods per plant basis. The results (Fig. 1) showed total number of flower and flower shedding per plant of different mungbean genotypes. It is evident from the present findings that different genotype behaved differently in the case of total number of flowers on per plant basis. Results showed that the highest number of flowers 125.93 per plant were observed in Dera-M which was at par to Sawat-1 and 12TM-03 with 116.60 and 112.80 flowers per plant, respectively. While 13TM-04, NM-98, NM-06, NM-11 and NM-16 behaved statistically at par from each other with 102.53, 100.93, 100.27, 100.13 and 98.13 flowers per plant, respectively. Minimum 85.47 flowers per plant were observed in AZRI-06 which was significantly different with all tested genotypes. The results (Fig. 1) also revealed that different genotypes had significant impact on number of flower shedding which may be due to thrips preference. Maximum 99.53 flower per plant shedding was observed in Dera-M which was significantly the highest than other tested genotypes followed by Sawat-1 with 87.67 flower shedding per plant. While minimum flower shedding per plant was recorded in AZRI-06 and 13TM-04 which was not significantly different from each other with 54.73 and 59.87 flowers shedding per plant, respectively. While genotypes NM-06 and NM-11 showed 74.47 and 74.27 flowers shedding per plant, respectively which was statistically at par with each other.

The results (Fig. 2) showed the total number of pods and deformed pods on per plant basis of different mungbean genotypes. It is clear from the results that utmost 34.60 pods per plant was observed in 13TM-04 which was statistically similar with NM-16 with 34.80 pods per plant followed by AZRI-06 with 28.73 pods per plant. Minimum 16.07 pods per plant were observed in NM-98 which was statistically at par with Sawat1 and Dera-M with 18.53 and 18.67 pods per plant. While 12TM-03, NM-11 and NM06 behaved statistically similar with $22.93,22.20$ and 21.20 total pods per plant, respectively. Figure 2 also showed that maximum deformed pods 4.93 and 4.33 were recorded in Dera-M and Sawat-1 and were statistically at par with each other. However minimum 2.07 deformed pods per plant were observed in 13TM-04 which was statistically at par with NM-16 and AZRI-06 with 2.33 and 2.60 deformed pods per plant, respectively. The genotypes NM-06, NM-98, 12TM-03 and NM-11 showed 3.27, 
3.07, 2.87 and 2.87 deformed pods per plant, respectively and were statistically at par from each other.

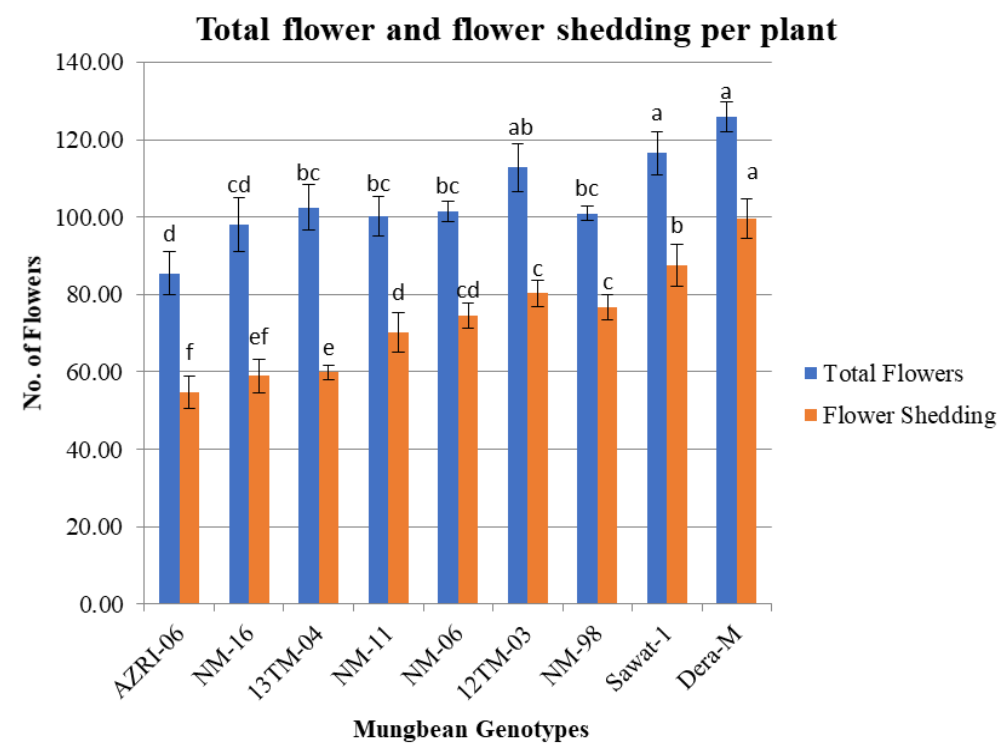

Figure 1. Response of different mungbean genotypes on number of flowers per plant

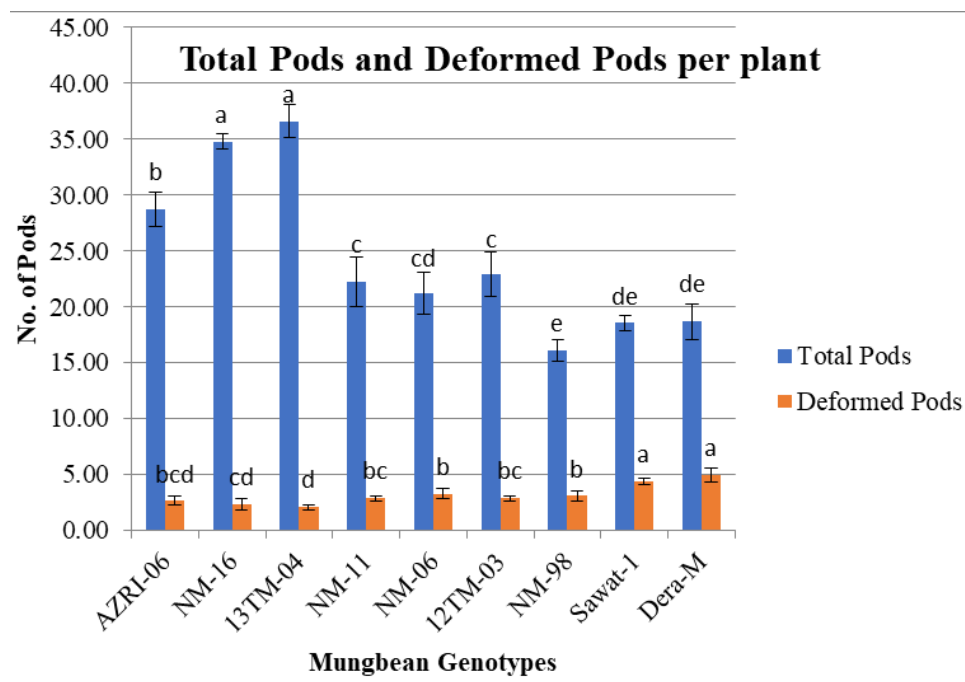

Figure 2. Response of different mungbean genotypes on number of pods per plant

\section{Role of different genotypes/varieties on the yield and the yield contributing characters}

The results (Table 3) showed the yield (kg/ha) and yield contributing characters of different mungbean genotypes/varieties which revealed that 13TM-04 showed maximum number 8.40 seeds per pod which was closely followed by NM-16 with 7.73 seeds per pod and were not significantly different from each other. While minimum 5.80 seeds per pod was observed in Dera-M which was not significantly different from NM-98 and Sawat-1 with 6.60 and 6.67 seeds per pod, respectively. While the genotypes AZRI-06, 12TM-03, NM-11 and NM-06 showed 7.47, 7.47, 7.07 and 6.80 seeds per pod, respectively and were not significantly different from each other. 
The outcome (Table 3) also revealed that maximum seed weight $4.77 \mathrm{~g}$ per plant was recorded in 13TM-04 which was not significantly different from NM-16 and AZRI-06 which showed 4.22 and $4.31 \mathrm{~g}$ seed weight per plant, respectively. Minimum $2.53 \mathrm{~g}$ seed weight per plant was recorded in Dera-M which was significantly different from the rest of the other tested genotypes followed by NM 98, Sawat-1 and NM-06 with $3.20,3.43$ and $3.70 \mathrm{~g}$ seed weight per plant, respectively, which were statistically at par with each other. The results (Table 3) also exposed that no significant difference exists among the, NM-16, 13TM-04, AZRI-06 and NM-11 genotypes of mungbean with 5.57, 5.51, 5.42 and $5.27 \mathrm{~g}$ maximum hundred seed weight, respectively. While minimum 3.80 hundred seed weight was recorded in Dera-M which was not significantly different with NM-98 and Sawat-1 with 4.04 and $4.31 \mathrm{~g}$ hundred seed weight respectively. The genotypes NM-06, 12TM-03 showed 4.72, $4.49 \mathrm{~g}$ hundred seed weight, respectively and did not differ significantly from each other.

The results (Table 3) also depicted that the per hectare yield significantly differ in the different selected mungbean genotypes. The highest yield $935 \mathrm{~kg} / \mathrm{ha}$ was recorded in NM-16 which was at par to $13 \mathrm{TM}-04$ which results $902.8 \mathrm{~kg} / \mathrm{ha}$ yield and were significantly different from all other selected genotypes of mungbean followed by AZRI-06 and NM-11 with yield 759.4 and $675.0 \mathrm{~kg} /$ ha yield, respectively. However minimum yield was recorded in Dera-M followed by NM-98 with 333.3 and $466.7 \mathrm{~kg} / \mathrm{ha}$ respectively which were significantly different from each other.

Table 3. Effect of different genotypes/varieties on the yield and the yield contributing characters of mungbean

\begin{tabular}{c|c|c|c|c|c}
\hline Treatments & No. of seed/pod & Seed weight/plant $(\mathbf{g})$ & $\mathbf{1 0 0}$ seed weight $(\mathbf{g})$ & $\begin{array}{c}\text { Yield/plot } \\
(\mathbf{g})\end{array}$ & Yield kg/ha \\
\hline AZRI-06 & $7.47 \mathrm{bc}$ & $4.22 \mathrm{ab}$ & $5.42 \mathrm{a}$ & $455.7 \mathrm{~b}$ & $759.5 \mathrm{~b}$ \\
NM-16 & $7.73 \mathrm{ab}$ & $4.31 \mathrm{ab}$ & $5.57 \mathrm{a}$ & $561.0 \mathrm{a}$ & $935.0 \mathrm{a}$ \\
$\mathbf{1 3 T M - 0 4}$ & $8.40 \mathrm{a}$ & $4.77 \mathrm{a}$ & $5.51 \mathrm{a}$ & $541.7 \mathrm{a}$ & $902.8 \mathrm{a}$ \\
NM-11 & $7.07 \mathrm{bc}$ & $4.08 \mathrm{bc}$ & $5.27 \mathrm{ab}$ & $405.0 \mathrm{c}$ & $675.0 \mathrm{c}$ \\
NM-06 & $6.80 \mathrm{c}$ & $3.70 \mathrm{bcd}$ & $4.72 \mathrm{bc}$ & $369.0 \mathrm{~cd}$ & $615.0 \mathrm{~cd}$ \\
$\mathbf{1 2 T M - 0 3}$ & $7.47 \mathrm{bc}$ & $3.98 \mathrm{bc}$ & $4.49 \mathrm{~cd}$ & $345.7 \mathrm{~d}$ & $576.1 \mathrm{~d}$ \\
NM-98 & $6.60 \mathrm{~cd}$ & $3.20 \mathrm{~d}$ & $4.04 \mathrm{de}$ & $280.0 \mathrm{e}$ & $466.7 \mathrm{e}$ \\
Sawat-1 & $6.67 \mathrm{~cd}$ & $3.43 \mathrm{~cd}$ & $4.31 \mathrm{cde}$ & $332.7 \mathrm{~d}$ & $554.2 \mathrm{~d}$ \\
Dera-M & $5.80 \mathrm{~d}$ & $2.53 \mathrm{e}$ & $3.80 \mathrm{e}$ & $200.0 \mathrm{f}$ & $333.3 \mathrm{f}$ \\
\hline LSD 0.05 & 0.88 & 0.66 & 0.56 & 42.77 & 71.28 \\
\hline
\end{tabular}

Means with the similar letter(s) are not significantly different from each other at $\mathrm{P}=0.05$

\section{Thrips population fluctuation versus climate factors, during 2018 and 2019}

The results (Table 4) revealed the thrips population fluctuation on per flower basis versus weather conditions. During 2018, the maximum thrips population were observed on the dates, the 26th June, with 5.099 thrips per flower at $43.50{ }^{\circ} \mathrm{C}, 26.88{ }^{\circ} \mathrm{C}$ and $25.19^{\circ} \mathrm{C}$ maximum, minimum and average temperature respectively and with $44.125 \%$ average R.H with rainfall nil which declined to 4.83 thrips per flower during the 30th June, 2018 and again increased to 5.07 thrips per flower on the date 04th July 2018 at $41.50{ }^{\circ} \mathrm{C}, 26.75^{\circ} \mathrm{C}$ and $34.13{ }^{\circ} \mathrm{C}$ maximum, minimum and average temperature respectively and with $58 \%$ average relative humidity. While during 2019 , the maximum 
5.11 thrips per flower were observed during the 26th, June 2019 which gradually increased to 5.22 thrips per flower during the 30th, Jun2 2019 and a peak population of 5.58 thrips per flower was observed during the 04th, July 2019 at $45.50{ }^{\circ} \mathrm{C}, 28.25$ and $36.88^{\circ} \mathrm{C}$ maximum, minimum and average temperature respectively and with $45.50 \%$ average R.H with rainfall nil.

Table 4. Data regarding meteorological observations on various weather factors and thrips population during 2018-19

\begin{tabular}{|c|c|c|c|c|c|c|c|}
\hline \multirow{2}{*}{ Sr.No. } & \multirow{2}{*}{ Date } & \multicolumn{3}{|c|}{ Temperature $^{\circ} \mathbf{C}$} & \multirow{2}{*}{$\begin{array}{c}\text { Average R.H } \\
(\%)\end{array}$} & \multirow{2}{*}{$\begin{array}{c}\text { Average } \\
\text { R.F }(\mathbf{m m})\end{array}$} & \multirow{2}{*}{$\begin{array}{c}\text { Average } \\
\text { thrips/flower }\end{array}$} \\
\hline & & Maximum & Minimum & Average & & & \\
\hline 1 & 14.06.18 & $\begin{array}{l}45.125 \\
(6.755)\end{array}$ & $\begin{array}{l}29.625 \\
(5.489)\end{array}$ & $\begin{array}{l}37.375 \\
(6.154)\end{array}$ & $\begin{array}{l}46.250 \\
(6.837)\end{array}$ & $\begin{array}{c}0.000 \\
(0.707)\end{array}$ & $\begin{array}{c}4.562 \\
(2.250)\end{array}$ \\
\hline 2 & 18.06.18 & $\begin{array}{l}43.500 \\
(6.633)\end{array}$ & $\begin{array}{l}28.500 \\
(5.385)\end{array}$ & $\begin{array}{l}36.000 \\
(6.042)\end{array}$ & $\begin{array}{l}47.500 \\
(6.928)\end{array}$ & $\begin{array}{c}0.000 \\
(0.707)\end{array}$ & $\begin{array}{c}4.763 \\
(2.294)\end{array}$ \\
\hline 3 & 22.06 .18 & $\begin{array}{l}41.750 \\
(6.500) \\
\end{array}$ & $\begin{array}{l}26.750 \\
(5.220) \\
\end{array}$ & $\begin{array}{l}34.250 \\
(5.895) \\
\end{array}$ & $\begin{array}{l}49.250 \\
(7.053) \\
\end{array}$ & $\begin{array}{c}2.500 \\
(1.732) \\
\end{array}$ & $\begin{array}{c}4.341 \\
(2.200) \\
\end{array}$ \\
\hline 4 & 26.06 .18 & $\begin{array}{l}43.500 \\
(6.633)\end{array}$ & $\begin{array}{l}26.875 \\
(5.232)\end{array}$ & $\begin{array}{l}35.188 \\
(5.974)\end{array}$ & $\begin{array}{l}44.125 \\
(6.680)\end{array}$ & $\begin{array}{l}0.000 \\
(0.707)\end{array}$ & $\begin{array}{c}5.099 \\
(2.366)\end{array}$ \\
\hline 5 & 30.06 .18 & $\begin{array}{l}39.750 \\
(6.344)\end{array}$ & $\begin{array}{l}26.000 \\
(5.148)\end{array}$ & $\begin{array}{l}32.875 \\
(5.777)\end{array}$ & $\begin{array}{l}54.500 \\
(7.416)\end{array}$ & $\begin{array}{c}0.000 \\
(0.707)\end{array}$ & $\begin{array}{c}4.831 \\
(2.309)\end{array}$ \\
\hline 6 & 04.07 .18 & $\begin{array}{l}41.500 \\
(6.481)\end{array}$ & $\begin{array}{l}26.750 \\
(5.220)\end{array}$ & $\begin{array}{l}34.125 \\
(5.884) \\
\end{array}$ & $\begin{array}{l}58.000 \\
(7.649)\end{array}$ & $\begin{array}{c}0.000 \\
(0.707)\end{array}$ & $\begin{array}{c}5.067 \\
(2.359)\end{array}$ \\
\hline 7 & 08.07 .18 & $\begin{array}{l}37.750 \\
(6.185) \\
\end{array}$ & $\begin{array}{l}25.875 \\
(5.136) \\
\end{array}$ & $\begin{array}{l}31.813 \\
(5.684) \\
\end{array}$ & $\begin{array}{l}55.750 \\
(7.500) \\
\end{array}$ & $\begin{array}{l}17.880 \\
(4.287) \\
\end{array}$ & $\begin{array}{c}4.028 \\
(2.128) \\
\end{array}$ \\
\hline 8 & 12.07.18 & $\begin{array}{l}45.250 \\
(6.764) \\
\end{array}$ & $\begin{array}{c}(29.375) \\
(5.466) \\
\end{array}$ & $\begin{array}{l}37.313 \\
(6.149) \\
\end{array}$ & $\begin{array}{l}48.250 \\
(6.982) \\
\end{array}$ & $\begin{array}{c}0.000 \\
(0.707) \\
\end{array}$ & $\begin{array}{c}4.999 \\
(2.345) \\
\end{array}$ \\
\hline 9 & 16.07.18 & $\begin{array}{l}42.500 \\
(6.557) \\
\end{array}$ & $\begin{array}{l}29.750 \\
(5.500) \\
\end{array}$ & $\begin{array}{l}36.125 \\
(6.052) \\
\end{array}$ & $\begin{array}{l}56.125 \\
(7.525) \\
\end{array}$ & $\begin{array}{c}0.000 \\
(0.707) \\
\end{array}$ & $\begin{array}{c}4.873 \\
(2.318) \\
\end{array}$ \\
\hline 10 & 20.07.18 & $\begin{array}{l}38.500 \\
(6.245) \\
\end{array}$ & $\begin{array}{l}26.000 \\
(5.148) \\
\end{array}$ & \begin{tabular}{|l|}
32.250 \\
$(5.723)$ \\
\end{tabular} & $\begin{array}{l}68.000 \\
(8.276) \\
\end{array}$ & $\begin{array}{l}13.500 \\
(3.742) \\
\end{array}$ & $\begin{array}{c}4.144 \\
(2.155) \\
\end{array}$ \\
\hline 11 & 24.07 .18 & $\begin{array}{l}40.500 \\
(6.403) \\
\end{array}$ & $\begin{array}{l}28.925 \\
(5.424) \\
\end{array}$ & \begin{tabular}{|l|}
34.713 \\
$(5.934)$ \\
\end{tabular} & $\begin{array}{l}68.125 \\
(8.284) \\
\end{array}$ & $\begin{array}{c}0.250 \\
(0.866) \\
\end{array}$ & $\begin{array}{c}4.503 \\
(2.237) \\
\end{array}$ \\
\hline 12 & 28.07.18 & $\begin{array}{l}39.000 \\
(6.285) \\
\end{array}$ & $\begin{array}{l}26.500 \\
(5.196) \\
\end{array}$ & $\begin{array}{l}32.750 \\
(5.766) \\
\end{array}$ & $\begin{array}{l}68.750 \\
(8.322) \\
\end{array}$ & $\begin{array}{c}4.875 \\
(2.318) \\
\end{array}$ & $\begin{array}{c}4.144 \\
(2.155) \\
\end{array}$ \\
\hline 13 & 01.08 .18 & $\begin{array}{l}39.500 \\
(6.325) \\
\end{array}$ & $\begin{array}{l}27.125 \\
(5.256) \\
\end{array}$ & \begin{tabular}{|l|}
33.313 \\
$(5.815)$ \\
\end{tabular} & $\begin{array}{l}60.625 \\
(7.818) \\
\end{array}$ & $\begin{array}{c}0.000 \\
(0.707) \\
\end{array}$ & $\begin{array}{c}4.273 \\
(2.185) \\
\end{array}$ \\
\hline 1 & 14.06 .19 & $\begin{array}{l}43.500 \\
(6.633) \\
\end{array}$ & $\begin{array}{l}28.870 \\
(5.419) \\
\end{array}$ & $\begin{array}{l}36.185 \\
(6.057) \\
\end{array}$ & $\begin{array}{l}46.500 \\
(6.856) \\
\end{array}$ & $\begin{array}{c}0.000 \\
(0.707) \\
\end{array}$ & $\begin{array}{c}4.867 \\
(2.317) \\
\end{array}$ \\
\hline 2 & 18.06 .19 & $\begin{array}{l}44.380 \\
(6.699) \\
\end{array}$ & \begin{tabular}{|l}
25.500 \\
$(5.099)$ \\
\end{tabular} & \begin{tabular}{|l|}
34.940 \\
$(5.953)$ \\
\end{tabular} & $\begin{array}{l}47.630 \\
(6.938) \\
\end{array}$ & $\begin{array}{c}0.500 \\
(1.000) \\
\end{array}$ & $\begin{array}{c}4.931 \\
(2.330) \\
\end{array}$ \\
\hline 3 & 22.06 .19 & $\begin{array}{l}38.250 \\
(6.225) \\
\end{array}$ & \begin{tabular}{|l|}
23.000 \\
$(4.848)$ \\
\end{tabular} & \begin{tabular}{|l|}
30.625 \\
$(5.579)$ \\
\end{tabular} & $\begin{array}{l}54.000 \\
(7.382) \\
\end{array}$ & $\begin{array}{c}3.750 \\
(2.062) \\
\end{array}$ & $\begin{array}{c}4.751 \\
(2.291) \\
\end{array}$ \\
\hline 4 & 26.06 .19 & $\begin{array}{l}40.380 \\
(6.394)\end{array}$ & $\begin{array}{l}26.130 \\
(5.160)\end{array}$ & $\begin{array}{l}33.255 \\
(5.810) \\
\end{array}$ & $\begin{array}{l}55.130 \\
(7.459)\end{array}$ & $\begin{array}{c}1.500 \\
(1.414)\end{array}$ & $\begin{array}{c}5.111 \\
(2.369)\end{array}$ \\
\hline 5 & 30.06 .19 & $\begin{array}{l}42.500 \\
(6.557)\end{array}$ & $\begin{array}{l}26.000 \\
(5.148)\end{array}$ & $\begin{array}{c}34.250 \\
(5.895) \\
\end{array}$ & $\begin{array}{l}52.880 \\
(7.306)\end{array}$ & $\begin{array}{c}0.750 \\
(1.118)\end{array}$ & $\begin{array}{c}5.223 \\
(2.392)\end{array}$ \\
\hline 6 & 04.07 .19 & $\begin{array}{l}45.500 \\
(6.782) \\
\end{array}$ & $\begin{array}{l}28.250 \\
(5.362)\end{array}$ & $\begin{array}{l}36.875 \\
(6.114) \\
\end{array}$ & $\begin{array}{l}45.500 \\
(6.782)\end{array}$ & $\begin{array}{c}0.000 \\
(0.707)\end{array}$ & $\begin{array}{c}5.583 \\
(2.466)\end{array}$ \\
\hline 7 & 08.07 .19 & $\begin{array}{l}39.130 \\
(6.295) \\
\end{array}$ & $\begin{array}{l}25.630 \\
(5.112) \\
\end{array}$ & $\begin{array}{l}32.380 \\
(5.734) \\
\end{array}$ & $\begin{array}{l}66.880 \\
(8.209) \\
\end{array}$ & $\begin{array}{c}8.250 \\
(2.958)\end{array}$ & $\begin{array}{c}4.763 \\
(2.294) \\
\end{array}$ \\
\hline
\end{tabular}




\begin{tabular}{c|l|l|c|c|c|c|c}
\hline \multirow{2}{*}{12.07 .19} & $\begin{array}{l}41.000 \\
(6.442)\end{array}$ & $\begin{array}{c}28.130 \\
(5.351)\end{array}$ & $\begin{array}{c}34.565 \\
(5.922)\end{array}$ & $\begin{array}{c}62.000 \\
(7.906)\end{array}$ & $\begin{array}{c}0.000 \\
(0.707)\end{array}$ & $\begin{array}{c}5.215 \\
(2.391)\end{array}$ \\
\hline \multirow{2}{*}{$\mathbf{9}$} & \multirow{2}{*}{16.07 .19} & 39.250 & 25.630 & 32.440 & 62.000 & 1.250 & 4.528 \\
& & $(6.305)$ & $(5.112)$ & $(5.739)$ & $(7.906)$ & $(1.323)$ & $(2.242)$ \\
\hline \multirow{2}{*}{$\mathbf{1 0}$} & \multirow{2}{*}{20.07 .19} & 39.130 & 26.750 & 32.940 & 65.250 & 0.550 & 4.215 \\
& & $(6.295)$ & $(5.220)$ & $(5.783)$ & $(8.109)$ & $(1.025)$ & $(2.171)$ \\
\hline \multirow{2}{*}{$\mathbf{1 1}$} & \multirow{2}{*}{24.07 .19} & 41.880 & 29.000 & 35.440 & 57.000 & 0.000 & 4.647 \\
& & $(6.510)$ & $(5.431)$ & $(5.995)$ & $(7.583)$ & $(0.707)$ & $(2.269)$ \\
\hline \multirow{2}{*}{$\mathbf{1 2}$} & \multirow{2}{*}{28.07 .19} & 37.950 & 28.000 & 32.975 & 66.630 & 0.250 & 4.338 \\
& & $(6.201)$ & $(5.339)$ & $(5.786)$ & $(8.193)$ & $(0.866)$ & $(2.200)$ \\
\hline \multirow{2}{*}{$\mathbf{1 3}$} & \multirow{2}{*}{01.08 .19} & 37.630 & 25.100 & 31.365 & 68.380 & 14.500 & 3.800 \\
& & $(6.175)$ & $(5.060)$ & $(5.645)$ & $(8.299)$ & $(3.873)$ & $(2.074)$ \\
\hline
\end{tabular}

Numbers in parenthesis are square root transformed

The data (Fig. 3) showed the thrips population and climate factors relationship at various dates of observations on collective basis for both research years 2018 and 2019. The outcome showed that a maximum thrips population (5.32/flower) was observed, on the $04^{\text {th }} \mathrm{July}$, at $43.50{ }^{\circ} \mathrm{C}, 27.50{ }^{\circ} \mathrm{C}$ and $35.50^{\circ} \mathrm{C}$ maximum, minimum and average temperature respectively with $51.75 \%$ relative humidity and with nil rainfall.

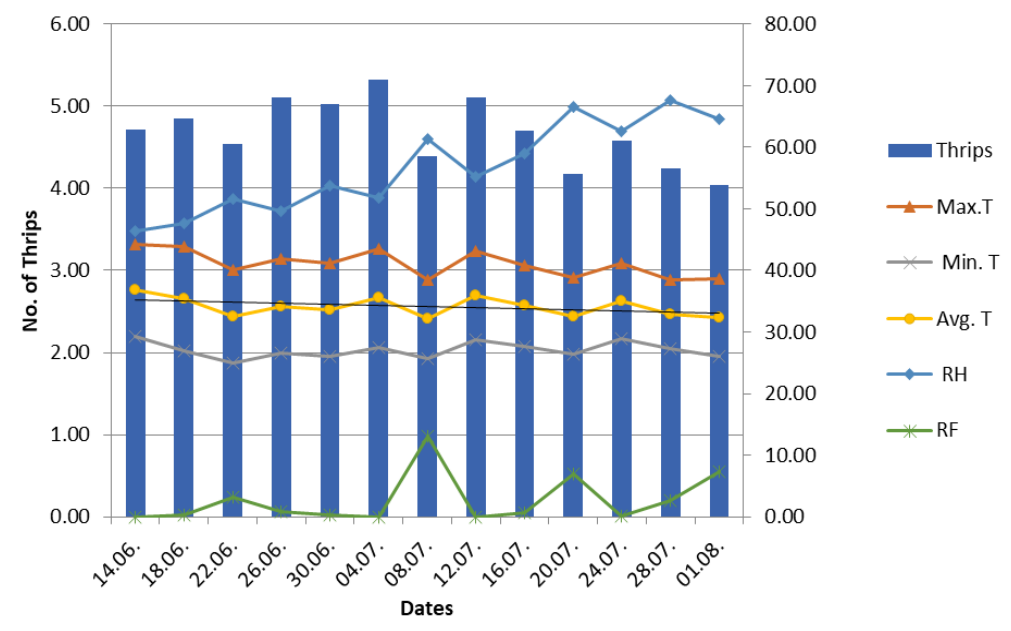

Figure 3. Thrips population fluctuation versus weather factors during 2018-19 on Cumulative basis

\section{Weather factors influence on thrips population}

The data (Table 5) revealed that during both research years 2018-19 maximum temperature and average temperature showed a positive and significant correlation with highly significant correlation with (r) value 0.90 and 0.668 respectively on cumulative basis, whereas average relative humidity and rainfall during both years showed negative and significant correlation with the thrips population with highly significant correlation with (r) value 0.732 and 0.750 respectively on collective basis. Whereas, non significant correlation was found with minimum temperature during both years and on cumulative basis. 
Table 5. Correlation coefficients (r) between thrips population per flower basis on mungbean, and various weather factors, during 2018 and 2019

\begin{tabular}{c|c|c|c}
\hline \multirow{2}{*}{ Weather factors } & \multicolumn{3}{|c}{ Per flower } \\
\cline { 2 - 4 } & $\mathbf{2 0 1 8}$ & $\mathbf{2 0 1 9}$ & Cumulative \\
\hline Max. temperature $\left({ }^{\circ} \mathbf{C}\right)$ & $0.715^{* *}$ & $0.747^{* *}$ & $0.90^{* *}$ \\
Min. temperature $\left({ }^{\circ} \mathbf{C}\right)$ & $0.413^{\mathrm{ns}}$ & $0.250^{\mathrm{ns}}$ & $0.285^{\mathrm{ns}}$ \\
Average temperature $\left({ }^{\circ} \mathbf{C}\right)$ & $0.644^{* *}$ & $0.633^{*}$ & $0.668^{* *}$ \\
Average relative humidity $(\boldsymbol{\%})$ & $-0.555^{*}$ & $-0.715^{* *}$ & $-0.732^{* *}$ \\
Average rain fall $(\mathbf{m m})$ & $-0.761^{* *}$ & $-0.553^{*}$ & $-0.750^{* *}$ \\
\hline
\end{tabular}

$*=$ Significant at $\mathrm{P} \leq 0.05 ; * *=$ Significant at $\mathrm{P} \leq 0.01 ; \mathrm{ns}=$ Non Significant

\section{The weather factors multiple effects on thrips population}

The results (Table 6 ) showed the weather factors multiple effects on per flower thrips population, during 2018 and 2019. According to findings during 2018 maximum contribution $(51.1 \%)$ was observed by maximum temperature in thrips population fluctuations followed by rainfall (14.8\%), average temperature $(3.0 \%)$ and average relative humidity $(1.4 \%)$. The $\mathrm{R}^{2}$ value was observed $70.3 \%$, when the effects of all climate factors on the per flower thrips fluctuation on mungbean was analyzed together for the year 2018 and the equation was good fitted. In 2019 maximum contribution was observed by maximum temperature $(55.8 \%)$ role in thrips per flower fluctuation of population followed by rainfall (4\%), average relative humidity $(1.2 \%)$ and average temperature $(1.0 \%)$. The $\mathrm{R}^{2}$ value of all climate factors for the year 2019 was observed $62.0 \%$, on the per flower thrips population fluctuation on mungbean and the equation was not fitted. The regression model regarding the impact of weather factors on thrips population in mungbean varieties revealed that maximum temperature was the most important factor which contributed maximum i.e. $51.1 \%$ and $55.8 \%$ role followed by rainfall which showed $14.8 \%$ and 4\% role during 2018 and 2019 respectively in the fluctuation of thrips population. The contribution of other weather factors to the thrips population variation was low (Table 5).

\section{Discussion}

Results of the present findings showed that out of twenty five tested genotypes against thrips infestation three genotypes namely 13TM-04, NM-16 and AZRI-06 showed resistant response in two years trial which are in confirmatory with Sreekanth et al. (2002) who reported that among thirty eight genotypes, four genotypes of mungbean constantly showed resistance to thrips namely LGG460, 480, 491 and 582 during 2000 and 2001. The results of the present findings are in accordance with Kooner and Malhi (2004) and with Brar et al. (2004) who obtained similar results although they used different mungbean varieties. The present results are in confirmatory with Rani et al. (2008) who found that out of 12 tested mungbean entries against thrips, less attack of thrips was recorded on MGG362 and MGG365 as compared to check MGG 295. The present findings are in agreement with Singh and Singh (2014) who screened 30 genotypes of mungbean against sucking insect pests including flower thrips. Minimum flower thrips infestation was recorded in ML-1628 and Pusa-1171 while maximum thrips infestation was recorded in BPMR-145, HUM-12 and Pusa-0672. In the present 
studies 13TM-04 constantly showed resistance against thrips attack which are in confirmatory with Indiati (2004) who investigated that mungbean cultivar MLG-716 constantly showed the resistance against thrips attack. The resistance of 13TM-04 may be due to antixenosis action.

Table 6. Multiple linear regression model/s along with $\left(R^{2}\right)$, regarding the impact of weather factors, on the per flower thrips population during 2018-19, on mungbean

\begin{tabular}{|c|c|c|c|c|c|}
\hline Regression equation & $\mathbf{R}^{2}$ & $100 R^{2}$ & $\begin{array}{c}\text { Percent } \\
\text { role }\end{array}$ & F value & P value \\
\hline \multicolumn{6}{|l|}{2018} \\
\hline$Y^{* *}=0.184+0.320 \mathrm{X}_{1}{ }^{* *}$ & 0.511 & 51.1 & 51.1 & 11.47 & 0.006 \\
\hline$Y^{*}=0.484+0.610 X_{1}-0.368 X_{2}$ & 0.541 & 54.1 & 3.0 & 5.89 & 0.020 \\
\hline$Y^{*}=-0.12+0.849 X_{1}-0.572 X_{2}+0.0349 X_{3}$ & 0.555 & 55.5 & 1.4 & 3.74 & 0.054 \\
\hline$Y^{*}=1.54+0.619 X_{1}-0.562 X_{2}+0.0117 X_{3}-0.0376 X_{4}$ & 0.702 & 70.3 & 14.8 & 4.73 & 0.030 \\
\hline \multicolumn{6}{|l|}{2019} \\
\hline$Y^{* *}=-0.266+0.398 X_{1}^{* *}$ & 0.558 & 55.8 & 55.8 & 13.89 & 0.003 \\
\hline$Y^{*}=-0.073+0.513 X_{1}-0.159 X_{2}$ & 0.568 & 56.8 & 1.0 & 6.58 & 0.015 \\
\hline$Y^{*}=0.89+0.335 X_{1}-0.066 X_{2}-0.0478 X_{3}$ & 0.580 & 58.0 & 1.2 & 4.15 & 0.042 \\
\hline$Y=1.19+0.503 X_{1}-0.333 X_{2}-0.017 X_{3}-0.0334 X_{4}$ & 0.620 & 62.0 & 4.0 & 3.26 & 0.073 \\
\hline
\end{tabular}

$*=$ Significant at $\mathrm{P} \leq 0.05 ; * *=$ Significant at $\mathrm{P} \leq 0.01 ; \mathrm{Y}$, Thrips population per flower; $\mathrm{X}_{1}=$ Maximum temperature $\left({ }^{\circ} \mathrm{C}\right) ; \mathrm{X}_{2}=$ Average temperature $\left({ }^{\circ} \mathrm{C}\right) ; \mathrm{X}_{3}=$ Average relative humidity $(\%)$, and $\mathrm{X}_{4}=$ Rainfall $(\mathrm{mm})$

Sucking insect pest populations fluctuation is significantly influenced by weather factors (Gogoi and Dutta, 2000; Murugan and Uthamasamy, 2001; Panickar and Patel, 2001). According to our findings maximum temperature has dominant role in thrips population fluctuation and is the most important abiotic factor which has highly significant and positive correlation while minimum temperature has positive and nonsignificant correlation which is in partial agreement with the Akram et al. (2013) who found that maximum and minimum temperature has strongly positive correlation with thrips population. Our outcomes are in agreement with the findings of Shivanna et al. (2011) who observed that with the rise in maximum temperature thrips population increases. Present results are not in agreement with Zala et al. (2014) who found that thrips has significantly negative association with maximum temperature. According to present findings rainfall and average relative humidity has a strong negative correlation on cumulative basis which confirm the results of the Patel et al. (2013) and Saini et al. (2017), which also affirmed the negative relation of thrips with rainfall. Our results confirmed the findings of Shahid et al. (2012) who observed that thrips population has negative relationship with rainfall and relative humidity. Our results are in agreement with the results of Akram et al. (2013) who found that with the increase in R.H, thrips population decreases because R.H has strong negative correlation. Our findings are not in confirmatory with Saleem et al. (2013) and Janu et al. (2017) who observed that thrips population has positive and noteworthy association with relative humidity and have no considerable relationship with maximum, minimum temperature and rainfall. Thrips population may be affected by rainfall by direct and indirect way (Morsello et al., 2014; Pereira et al., 2007; Semeao et al., 2012). Our findings showed that rainfall have significant and negative correlation during 2018, 2019 and on cumulative basis 
which is in confirmatory with the finding of Sedaratian et al. (2010) who found that thrips have negative association with rainfall and relative humidity. Rainfall causes mortality of insect due to the mechanical impact of droplets which wash small insects down onto the soil and the indirectly rainfall increases the humidity (Semeao et al., 2012). Similarly, Barbosa et al. (2019) found a negative impact of rainfall on thrips population in multiple regression analysis.

\section{Conclusion}

The outcomes of the current findings identified various partially resistant cultivars namely 13TM-04, NM-16 and AZRI-06 against thrips attack. Moreover, to evolve new varieties these genotypes can be used as a source of resistance by the plant breeders for breeding programs. Thrips population in mungbean is influenced by different weather factors in which maximum temperature and rainfall play a significant role. During cropping season the knowledge about thrips dynamics helps in manipulating suitable pest managing strategy. The data generated in the present study will be helpful in the establishment of predictive models of thrips attack on mungbean in relation to weather factors. However, further work is needed to investigate the different sowing times and new varieties which may perform well in the prevalent environmental conditions against thrips. Moreover, role of physio-morphological and chemical plant characters on different mungbean genotypes should be studied.

Acknowledgments. Sincere thanks to the Higher Education Commission, Islamabad for providing the financial grant and Arid Zone Research Institute (AZRI) Bhakkar for providing research facilities.

Conflict of interests. The authors declare that they have no conflict(s) of interests.

\section{REFERENCES}

[1] Akram, M., Hafeez, F., Farooq, M., Arshad, M., Hussain, M., Ahmed, S., Zia, K., Ali Khan, H. A. (2013): A case to study population dynamics of Bemisia tabaci and Thrips tabaci on Bt and non-Bt cotton genotypes. - Pakistan Journal of Agricultural Sciences 50(4): 617-623.

[2] Ali, A., Farooq, A. (2019): Evaluation of population dynamics of sucking pests on Bt and non Bt cotton cultivars. - Pakistan Journal of Zoology 51(3): 1093-1093.

[3] Anjum, M., Ahmed, Z., Rauf, C. A. (2006): Effect of Rhizobium inoculation and nitrogen fertilizer on yield and yield components of mungbean. - International Journal of Agriculture and Biology (Pakistan) 8(2): 238-240.

[4] Barbosa, B. G., Sarmento, R. A., Pereira, P. S., Pinto, C. B., de Oliveira Lima, C. H., da Silva Galdino, T. V., Santos, A. A., Picanço, M. C. (2019): Factors affecting thrips (Thysanoptera: Thripidae) population densities in watermelon crops. - Florida Entomologist 102(1): 10-15.

[5] Brar, J., Singh, I., Singh, S., Bains, T. (2004): SML 668: A new early maturing and high yielding variety of summer mungbean. - Journal of Research 41(1): 174-174.

[6] Da Silva, R. S., Kumar, L., Shabani, F., Da Silva, E. M., da Silva Galdino, T. V., Picanço, M. C. (2017): Spatio-temporal dynamic climate model for Neoleucinodes elegantalis using CLIMEX. - International Journal of Biometeorology 61(5): 785-795.

[7] Gogoi, I., Dutta, B. (2000): Seasonal abundance of cotton jassid, Amrasca biguttula biguttula (Ishida) on okra. - J. Agric. Sci. Soc. North-East India 13: 22-26. 
[8] Herms, D. A. (2004): Using Degree-Days and Plant Phenology to Predict Pest Activity. In: Krischik, V., Davidson, J. (eds.) IPM (Integrated Pest Management) of Midwest Landscapes. Minnesota Agricultural Experiment Station Publication, Saint Paul, MN, pp. 49-59.

[9] Hossain, M., Ferdous, J., Sarkar, M., Rahman, M. (2004): Insecticidal management of thrips and pod borer in mungbean. - Bangladesh J. Agril. Res 29(3): 347-356.

[10] Indiati, S. (2004): Screening and resistance mechanism of mungbean MLG-716 to thrips. - Jurnal Litbang Pertanian 23(3): 100-106.

[11] Iqbal, J., Ashfaq, M., ul Hasan, M., Sagheer, M., Nadeem, M. (2010): Influence of abiotic factors on population fluctuation of leaf hopper, Amrasca biguttula biguttula (Ishida) on Okra. - Pakistan Journal of Zoology 42(5).

[12] Janu, A., Dahiya, K., Jakhar, P. (2017): Population dynamics of thrips, Thrips tabaci Lindemann in American cotton (Gossypium hirsutum). - Int. J. Curr. Microbiol. App. Sci 6(7): 203-209.

[13] Kaul, J., Singh, K., Sekhon, H. (1976): The amount of flower shedding in some kharif pulses. - The Journal of Agricultural Science 86(1): 219-219.

[14] Kooner, B., Malhi, B. (2004): Sources of resistance to bean thrips, Megalurothrips distalisin summer mungbean. - Abstr No. AV. 5. Souvenir and Abstr. 7th Punjab Sci. Congr., GNDU, Amritsar.

[15] Malik, B., Nazir, S., Bashir, E. (1994): Grain Legumes: Crop Production. - National Book Foundation, Islamabad, pp. 303-304.

[16] Morsello, S. C., Groves, R. L., Nault, B. A., Kennedy, G. G. (2014): Temperature and precipitation affect seasonal patterns of dispersing tobacco thrips, Frankliniella fusca, and onion thrips, Thrips tabaci (Thysanoptera: Thripidae) caught on sticky traps. Environmental Entomology 37(1): 79-86.

[17] Murugan, M., Uthamasamy, S. (2001): Dispersal behaviour of cotton whitefly Bemisia tabaci (Genn.) under cotton based gardenland agro ecosystem of Coimbatore, Tamil Nadu. - Madras Agricultural Journal 88(1/3): 1-6.

[18] Panickar, B., Patel, J. (2001): Population dynamics of different species of thrips on chilli, cotton and pigeonpea. - Indian Journal of Entomology 63(2): 170-175.

[19] Patel, H., Patel, K., Chawda, S., Siddhapara, M. (2013): Impact of abiotic factors on population dynamics of Insect pest of jatropha in south Gujarat. - The Bioscan 8(1): 9193.

[20] Pedigo, L. P. (2004): Entomology and Pest Management. 4th Ed. - Prentice Hall of India, New Delhi, pp. 175-210.

[21] Pereira, E. J. G., Picanço, M. C., Bacci, L., Della Lucia, T. M. C., Silva, É. M., Fernandes, F. L. (2007): Natural mortality factors of Leucoptera coffeella (Lepidoptera: Lyonetiidae) on Coffea arabica. - Biocontrol Science and Technology 17(5): 441-455.

[22] Rani, C. S., Eswari, K., Sudarshanam, A. (2008): Field screening of greengram (Vigna radiata L.) entries against thrips (Thrips palmi) and spotted pod borer (Maruca vitrata). Journal of Research Angrau 36(2/3): 17-22.

[23] Ratnasekera, D., Subhashi, A. (2015): Morpho-physiological response of selected mungbean (Vigna radiata L.) Sri Lanka genotypes to drought stress. - Journal of Agriculture Search 2(3): 162-166.

[24] Rosado, J. F., Picanço, M. C., Sarmento, R. A., Da Silva, R. S., Pedro-Neto, M., Carvalho, M. A., Erasmo, E. A., Silva, L. C. R. (2015): Seasonal variation in the populations of Polyphagotarsonemus latus and Tetranychus bastosi in physic nut (Jatropha curcas) plantations. - Experimental and Applied Acarology 66(3): 415-426.

[25] Sagar, T., Venkatarao, P., Gautam, C. (2017): Varietal screening of mungbean cultivars for resistance/tolerance against insect pest under Terai Agro ecological zone of West Bengal. - International Journal of Plant Protection 10(1): 7-13. 
[26] Saini, A., Ahir, K., Rana, B., Kumar, R. (2017): Population dynamics of sucking pests infesting chilli (Capsicum annum L.). - Journal of Entomology and Zoology Studies 5(2): 250-252.

[27] Saleem, M. W., Ahmed, S., Wakil, W., Sahi, S. T. (2013): Resistance to thrips, Thrips tabaci in Bt cotton genotypes in Punjab, Pakistan, based on population dynamics and plant morpho-chemical properties. - Int. J. Agric. Biol 15: 331-336.

[28] Sedaratian, A. a., Fathipour, Y., Talebi, A., Farahani, S. (2010): Population density and spatial distribution pattern of Thrips tabaci (Thysanoptera: Thripidae) on different soybean varieties. - Journal of Agricultural Science and Technology 12(3): 275-288.

[29] Semeao, A. A., Martins, J. C., Picanço, M. C., Bruckner, C. H., Bacci, L., Rosado, J. F. (2012): Life tables for the guava psyllid Triozoida limbata in southeastern Brazil. BioControl 57(6): 779-788.

[30] Shahid, M. R., Farooq, J., Mahmood, A., Ilahi, F., Riaz, M., Shakeel, A., Petrescu-Mag, I. V., Farooq, A. (2012): Seasonal occurrence of sucking insect pest in cotton ecosystem of Punjab, Pakistan. - Advances in Agriculture \& Botanics 4(1): 26-30.

[31] Shivanna, B., Gangadhara, B., Basavaraja, M., Nagaraja, R., Kalleswara, C., Karegowda, C. (2011): Impact of abiotic factors on population dynamics of sucking pests in transgenic cotton ecosystem. - International Journal of Natural Sciences 2: 72-74.

[32] Shuaib, M. (2004): Ecosystem analysis of vegetable crop health for sustainable production. - Thesis, University of Arid Agriculture, Rawalpindi.

[33] Singh, S. K., Singh, P. (2014): Screening of mungbean (Vigna radiata) genotypes against major insects. - Current Advances in Agricultural Sciences 6(1): 85-87.

[34] Sinha, S. K. (1977): Food Legumes: Distribution, Adaptability and Biology of Yield. FAO, Rome.

[35] Soni, R., Dhakad, N. (2016): Seasonal dynamics of Thrips tabaci (Lindemann) and their correlation with weather parameters on transgenic Bt cotton. - International Journal of Advanced Research 4(8): 1486-1488.

[36] Sreekanth, M., Sreeramulu, M., Rao, R. D., Babu, B. S., Babu, T. R. (2002): Evaluation of greengram genotypes (Vigna radiata L. Wilczek) for resistance to Thrips palmi Karny and peanut bud necrosis virus. - Indian Journal of Plant Protection 30(2): 109-114.

[37] Steel, R. G. D., Torrie, J. H. (1997): Principles and Procedures of Statistics with Special Reference to the Biological Sciences. - McGraw Hill Book Co. Inc., New York.

[38] Steel, R. G. D., Torrie, J. H., Dickey, D. A. (1997): Principles and Procedures of Statistics: A Biometrical Approach. 3rd Ed. - WCB MeGraw Hill Companies, Inc., New York.

[39] Yaqub, M., Mahmood, T., Akhtar, M., Iqbal, M. M., Ali, S. (2010): Induction of mungbean [Vigna radiata (L.) Wilczek] as a grain legume in the annual rice-wheat double cropping system. - Pak. J. Bot. 42(5): 3125-3135.

[40] Zala, M., Patel, C., Bharpoda, T. (2014): Impact of sowing periods on incidence of sucking pests and their relation to weather parameters in Bt cotton. - Ecoscan 6: 345-354. 